\title{
Guiding Mesenchymal Stem Cells into Myelinating Schwann Cell-like Phenotypes by Using Electrospun Core-Sheath Nanoyarns
}

Shaohua $\mathrm{Wu}^{\dagger, \S}$, Shilei Ni ${ }^{\ddagger}$, Xiping Jiang ${ }^{\dagger}$, Mitchell A. Kuss ${ }^{\dagger}$, Hanjun Wang ${ }^{\perp}$, Bin Duan*,†,\#,\|

${ }^{\dagger}$ Division of Cardiology, Department of Internal Medicine; Mary \& Dick Holland Regenerative Medicine Program, University of Nebraska Medical Center, Omaha, NE, 68198, USA

§College of Textiles \& Clothing, Qingdao University, Qingdao, 266071, China

Department of Neurosurgery, Qilu Hospital of Shandong University and Institute of Brain and Brain-Inspired Science, Shandong University, Jinan, 250012, China

${ }^{\perp}$ Department of Anesthesiology, University of Nebraska Medical Center, Omaha, NE, 68198, USA

\#Department of Surgery, College of Medicine, University of Nebraska Medical Center, Omaha, NE, 68198, USA

"Department of Mechanical and Materials Engineering, University of Nebraska-Lincoln, Lincoln, NE, 68588, USA

*Corresponding author: E-mail address: bin.duan@unmc.edu; Phone: (402) 559-9637

KEYWORDS: core-sheath yarn, myelination, Schwann cell-like differentiation, cell migration, nerve regeneration 


\begin{abstract}
Nerve guidance conduits (NGC)-infilling substrates have been reported to facilitate the regeneration of injured peripheral nerves $(\mathrm{PN})$, especially for large nerve gaps. In this study, longitudinally oriented electrospun core-sheath nanoyarns (csNY), consisting of a polylactic acid microfiber core and an electrospun nanofiber sheath, were fabricated for potential PN tissue engineering applications. Our novel csNY displayed a well-aligned nanofibrous surface topography, resembling the ultrastructure of axons and fascicles of a native PN system, and they also provided a mechanically stable structure. The biological results showed that the csNY significantly enhanced the attachment, growth, and proliferation of human adipose derived mesenchymal stem cells (hADMSC) and also promoted the migration, proliferation, and phenotype maintenance of rabbit Schwann cells (SC). Our csNY notably increased the differentiation capability of hADMSC into SC-like cells (hADMSC-SC), in comparison with a 2D tissue culture polystyrene plate. More importantly, when combined with the appropriate induction medium, our csNY promoted hADMSC-SC to express high levels of myelination-associated markers. Overall, this study demonstrates that our csNY have great potential to serve as not only ideal in vitro culture models for understanding SC-axon interaction and SC myelination, but also as promising NGC-infilling substrates for $\mathrm{PN}$ regeneration applications.
\end{abstract}




\section{INTRODUCTION}

Peripheral nerve $(\mathrm{PN})$ injuries are a common consequence of traumatic injuries and affect $2.8 \%$ $5 \%$ of trauma patients, frequently leading to life-long disability. ${ }^{1,2}$ Over 200,000 peripheral nerve repair procedures are performed annually in the United States. ${ }^{3,4}$ For large PN gap injuries $(>10$ $\mathrm{mm})$, nerve grafts must be employed to augment the severe injuries in order to improve the healing rates. ${ }^{5,6}$ Although autologous nerve grafts are considered to be the gold standard for transected peripheral nerve defects greater than approximately $30 \mathrm{~mm}$, this strategy is still far from ideal because of limited supply, mismatches in nerve diameter, and donor site morbidity, such as the loss of native function and neuroma formation. ${ }^{7,8}$ Allografts and xenografts have also been employed, but concerns persist over the potential for immunological rejection, disease transmission, ethical issues, inferiority to autografts, and inconsistent and unsatisfactory results. ${ }^{9,10}$ There is still a huge need for generating biological augmentation strategies that develop artificial nerve grafts and promote functional regeneration for damaged PN, especially for large PN defects.

As a promising and attractive approach, engineered nerve grafts, normally in conduit form with an empty lumen, have been developed to mechanically bridge the gap and guide nerve regeneration. ${ }^{11-13} \mathrm{~A}$ wide variety of manufacturing techniques and biomaterials have been implemented for preparing nerve guidance conduits (NGC) with various complex configurations, such as fiber-like nanostructures, ${ }^{14,15}$ micro-grooves, ${ }^{16,17}$ and multi-channels. ${ }^{18,19}$ Some of them have already been approved by US Food and Drug Administration for human use. ${ }^{20,21}$ However, they are not recommended for gaps larger than $30 \mathrm{~mm}$ in the clinic because NGC alone usually 
cannot perform satisfactorily, showing insufficient axon regeneration and limited functional recovery for large defects. This is most likely due to the suboptimal ultrastructure and the lack of supportive intraluminal microenvironments. ${ }^{22,23}$ Although some intraluminal fillings, such as aligned fibers, ${ }^{24,25}$ spongy matrix, ${ }^{26,27}$ and hydrogels, ${ }^{28,29}$ have been employed to provide guidance cues for Schwann cell (SC) migration and axonal regeneration, they still poorly replicate the hierarchical and multiscale architectures in native PN. This inevitably results in reduced cellular activity and inferior regeneration outcomes. Thus, more effort is needed to improve the fabrication of the intraluminal filling in order to better control the microenvironment inside the conduit so that it promotes SC migration, axonal regrowth, reconnection, and functional repair.

Following injury, SC, which are the principal glial cells of the PN system that produce the myelin sheath for insulating and supporting axons, play a crucial role in multiple processes that lead to secondary PN degeneration, remyelination, and further regeneration. ${ }^{30,31}$ Since native primary SC with high enough quality and quantity are not sufficiently available, stem cell therapy in PN injury is a promising therapeutic clinical strategy. ${ }^{32,33}$ Several types of stem cells, including neural stem cells, ${ }^{34-35}$ skin derived precursors, ${ }^{36,37}$ umbilical cord derived mesenchymal stem cells (MSC), ${ }^{38,39}$ bone marrow derived MSC, ${ }^{40,41}$ and adipose derived MSC (ADMSC), ${ }^{42,43}$ have been used and proven to have positive effects on PN regeneration. The stem cell-based approaches tend to establish a more favorable environment for preventing SC senescence, facilitating endogenous SC migration, and promoting remyelination and reinnervation. ${ }^{44}$ Among various stem cells, ADMSC are a safe and effective therapeutic option, since they are multipotent, readily available, 
have low immunogenicity, and have a high capacity to differentiate into SC-like cells. ${ }^{45,46}$ Several studies have demonstrated that ADMSC alone or an ADMSC and SC co-culture better promoted motor recovery and nerve regeneration, compared to acellular NGC.47-49 Although in vitro ADMSC differentiation into SC has been explored, most studies have relied on 2D tissue culture polystyrene (TCPS) plates, which is not an ideal method due to a lack of cell-matrix interactions. ${ }^{50-}$ ${ }^{52}$ Moreover, the 2D plate culture requires extra steps for passaging the cells before utilization. Therefore, it is significantly important to develop nanofiber-based intraluminal fillings that can resemble the physiological conditions of native ECM and be utilized as ready-to-implant grafts. Ideally, such grafts can also control ADMSC differentiation into SC and even myelinating SC.

In this work, we employed a modified electrospinning device to fabricate core-sheath nanoyarns (csNY) consisting of a microfiber core and a nanofiber sheath for potential application in PN regeneration. We hypothesized that $\operatorname{csNY}$ with an aligned nanofibrous topographies and mechanically-stable structures improve ADMSC differentiation into SC like cells (ADMSC-SC). We systematically investigated the cell morphology, adhesion, migration, and proliferation of human ADMSC (hADMSC) and rabbit SC (rSC) when seeded on various fibrous yarns. We further compared the differentiation efficiency of hADMSC into SC-like cells on different matrices and induced hADMSC differentiation into myelinating SC-like cells on csNY. The csNY with myelinating hADMSC-SC are promising intraluminal fillers in NGC for long gap PN repair. 


\section{MATERIALS AND METHODS}

\subsection{Fabrication of nanoyarns with a core-sheath structure}

Gelatin (Gel, from bovine skin, Sigma) was modified through the reaction of methacrylic anhydride (Sigma) in deionized water to generate methacrylated gelatin (MeGel), according to the procedures reported previously. ${ }^{53,54}$ The methacrylation degree was determined to be roughly $61.1 \%$ for the as-obtained MeGel. ${ }^{54}$ Poly (lactic-co-glycolic) acid (PLGA, 50/50 LA/GA, Corbion Purac), MeGel, and MeGel/PLGA (50/50 w/w) were dissolved in hexafluoro-2-propanol (HFIP, Acros Organics) with an overall polymer concentration of $10 \%(\mathrm{w} / \mathrm{v})$. Then they were stirred and mixed overnight to obtain a homogeneous electrospinning solution. A modified electrospinning system was utilized to spin the three different polymer solutions to form csNY (Figure 1A). A solution supply was utilized to continuously deliver polymer solutions into two face-to-face $18 \mathrm{G}$ metal needles ( $20 \mathrm{~cm}$ distance between the two needles) at a flow rate of $1 \mathrm{~mL} / \mathrm{h}$. A high voltage supply was employed to apply positive and negative voltages $( \pm 12 \mathrm{kV})$ on the two needles respectively, and polymeric nanofibers with opposite charges were generated from these two needles. A yarn-supply roll and one set of tension devices were used to continuously convey polylactic acid (PLA) microfiber yarns (MY) (Shaoxing Zhongfangyuan Co., Ltd, China). A rotating neutral metal disc (NMD, $250 \mathrm{r} / \mathrm{min}$ ) and a static neutral hollow metal rod (NHMR), placed oppositely ( $7 \mathrm{~cm}$ distance between NMD and NHMR), were utilized to collect and coat polymeric nanofibers onto PLA MY and further process them into NY. The obtained csNY, consisting of a PLA microfiber core and a MeGel, PLGA, or MeGel/PLGA nanofiber sheath 
(denoted as MeGel csNY, PLGA csNY or MeGel/PLGA csNY), were passed through the inner part of NHMR and continuously gathered on a collecting roll at a rotating speed of $2 \mathrm{~m} / \mathrm{min}$. The as-electrospun MeGel and MeGel/PLGA csNY were further immersed into 5\% w/v 2-hydroxy1(4-(hydroxyethyl)phenyl)-2-methyl-1-propanone (Irgacure 2959; CIBA Chemicals) ethanol solution and subsequently exposed to a $365 \mathrm{~nm}$ UV light (OmniCure S2000 UV lamp, Lumen Dynamics) for 5 minutes at room temperature to crosslink and stabilize the MeGel component in the csNY. A vacuum chamber was employed to desiccate all of the different csNY before further characterization.

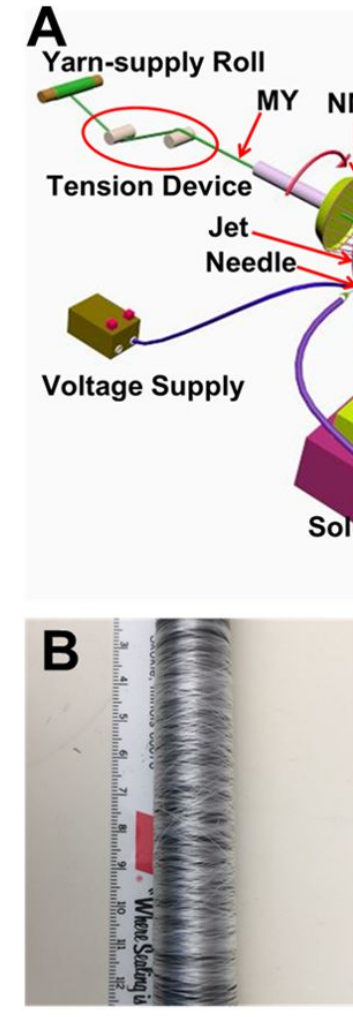

PLA MY

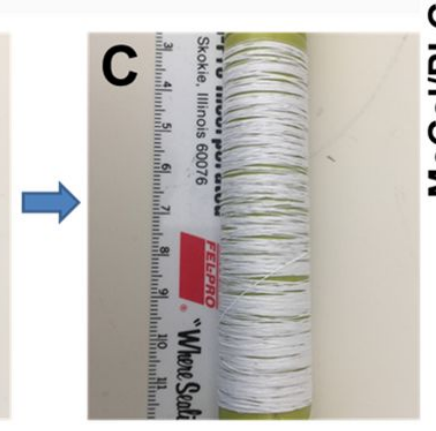

PLGA csNY
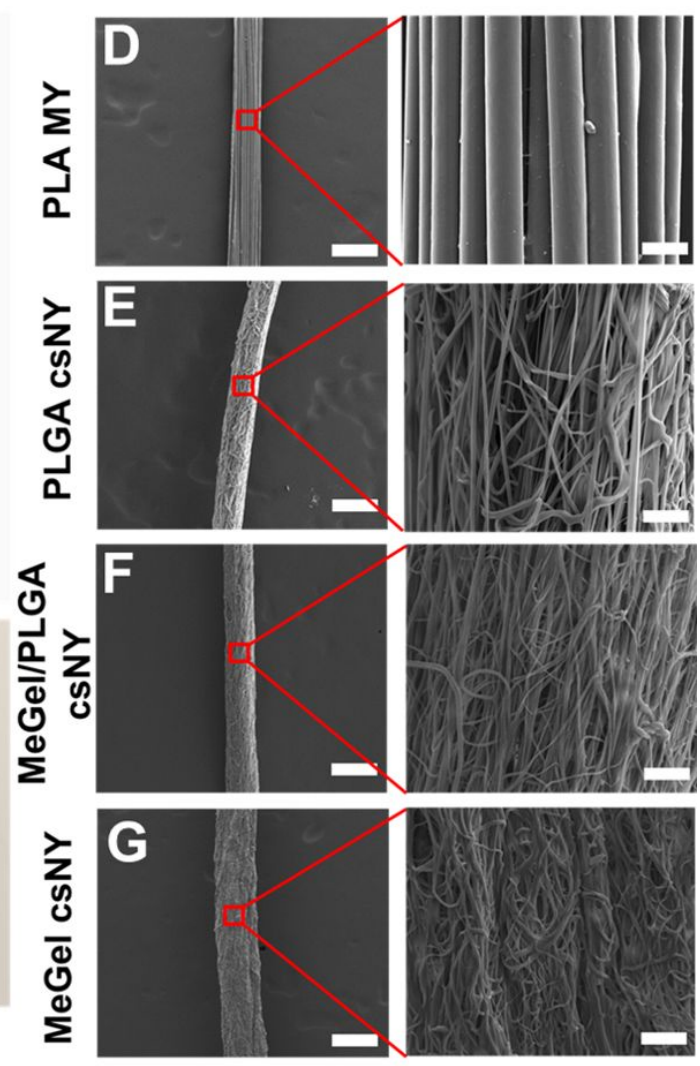
Figure 1. Fabrication of csNY. (A) Schematic illustration of the modified electrospinning system for coating electrospun nanofibers on the surface of PLA MY to generate csNY. (B) Photograph of a PLA MY package. (C) Photograph of an as-prepared PLGA csNY package employing PLA MY as core yarn. SEM image of the pristine PLA MY (D), the obtained PLGA csNY (E), MeGel/PLGA csNY (F), and MeGel csNY (G). Scale bars: $200 \mu \mathrm{m}$ for left panel; $20 \mu \mathrm{m}$ for right panel.

\subsection{Physical characterization of the yarn materials}

A scanning electron microscope (SEM, FEI Quanta 200) was utilized to conduct morphological assessments of different yarn samples. Prior to obtaining the SEM images, a thin layer of gold was sputter-coated onto the material samples. The fiber and yarn diameters and the fiber orientation angle distribution (relative to the yarn's radial direction) for the different yarn samples were measured using Image $\mathbf{J}$ software $(\mathrm{NIH})$. We employed a Fourier transform infrared (FTIR) spectrometer (Nicolet ${ }^{\mathrm{TM}}$ iS50, Thermo Fisher Scientific) in attenuated total reflectance mode (ATR) to investigate the functional groups of all the as-fabricated csNY. FTIR spectra were obtained in the $4000-500 \mathrm{~cm}^{-1}$ region using 16 scans and $1 \mathrm{~cm}^{-1}$ resolution at room temperature. The tensile mechanical properties of the different yarn materials were examined using an XQ-2 fiber strength tester (Shanghai Lipu Institute of Applied Science and Technology, Shanghai, China) with a gauge length of $10 \mathrm{~mm}$ and a constant rate of $10 \mathrm{~mm} / \mathrm{min}$ until failure occurred. We 
calculated the Young's modulus of the yarns from the initial 5\%-10\% strain region of stress-stain curves. The failure load, ultimate tensile strength, and strain to failure were also determined.

\subsection{HADMSC seeding and culture}

Primary hADMSC were purchased from Lonza and cultured in a growth medium (GM) consisting of Dulbecco's modified Eagle's medium/F12 (DMEM/F12, Invitrogen) medium, 10\% fetal bovine serum (FBS, Gibco), and 1\% penicillin/streptomycin (P/S, Invitrogen). HADMSC were used at passages 4-6. Before cell seeding, the yarn samples were trimmed into $2 \mathrm{~cm}$ long segments and sterilized in $70 \%(\mathrm{v} / \mathrm{v})$ ethanol overnight. After washing them with sterilized phosphate buffered saline (PBS) solution, they were further submerged in GM overnight. The hADMSC were seeded at a density of $5 \times 10^{3}$ cells per yarn. All cell incubation experiments in this study were conducted in $5 \% \mathrm{CO}_{2}$ at $37{ }^{\circ} \mathrm{C}$, and the medium was changed every 2 days.

\subsection{HADMSC differentiation into hADMSC-SC}

HADMSC were seeded onto MeGel/PLGA NY or 2D TCPS plates and cultured in GM for 1 day, and then they were induced by using a series of induction media (IM), according to the following procedures. IM-I, consisting of DMEM/F12, $1 \% \mathrm{P} / \mathrm{S}$, and $1 \mathrm{mM} \beta$-mercaptoethanol (Sigma), was employed to culture seeded hADMSC for $24 \mathrm{~h}$. After washing with sterilized PBS solution, the medium was replaced with IM-II, consisting of DMEM/F12, 10\% FBS, $1 \% \mathrm{P} / \mathrm{S}$, and $35 \mathrm{ng} / \mathrm{ml}$ all trans-retinoic acid (Sigma), and cultured for $72 \mathrm{~h}$. After washing with sterilized PBS solution, hADMSC were treated and further induced with IM-III, consisting of DMEM/F12, 10\% FBS, 1\% $\mathrm{P} / \mathrm{S}, 5.7 \mu \mathrm{M}$ forskolin (Sigma), $200 \mathrm{ng} / \mathrm{ml}$ recombinant human heregulin- $\beta 1$ (PeproTech), $10 \mathrm{ng} / \mathrm{ml}$ 
basic fibroblast growth factor (bFGF, PeproTech), and $5 \mathrm{ng} / \mathrm{ml}$ recombinant human platelet derived growth factor-AA (PDGF-AA, PeproTech), for 10 days. For further differentiation into myelinating SC-like cells, the obtained, differentiated hADMSC-csNY constructs were divided into two groups for subsequent processing: (i) Differentiated hADMSC-csNY constructs were maintained in IM-III for another 14 days; (ii) Differentiated hADMSC-csNY constructs were further induced by utilizing IM-IV (neural progenitor medium), consisting of neurobasal medium (Hyclone), DMEM/F12 medium (Hyclone) (1:1), 1× B27, $1 \times \mathrm{N} 2$, bFGF $(20 \mathrm{ng} / \mathrm{mL})$, and 1\% P/S, for 14 days.

\subsection{RSC harvest and migration characterization}

Sciatic nerves from three adult New Zealand white rabbits were excised, stripped of the epineurium, and cut into $2 \mathrm{~mm}$ fragments. The animals were euthanized subjects from another research protocol, which was carried out in accordance with recommendations in the Guide for the Care and Use of Laboratory Animals from the National Research Council and the National Institutes of Health and also in accordance with the Animal Welfare Act of the United States. The animal protocol was approved by the Institutional Animal Care and Use Committee (IACUC) of the University of Nebraska Medical Center (UNMC). The fragments were enzymatically digested in $0.25 \%$ trypsin and $1 \%$ collagenase for $1 \mathrm{~h}$ at $37^{\circ} \mathrm{C}$. Then the mixture was centrifuged and resuspended in DMEM medium (Hyclone) with 10\% FBS and 1\% P/S. Then the resuspended cell mixture was added to petri dishes. After $24 \mathrm{~h}$ of incubation, $10 \mathrm{mM}$ cytosine arabinoside was added to the dishes, and they were incubated for another $48 \mathrm{~h}$ in order to remove fibroblasts. Subsequently, medium 
consisting of DMEM, 10\% FBS, and 1\% P/S was utilized to passage and culture purified rSC, which were used at passages 4-6. The rSC migration behavior on MeGel/PLGA NY was characterized by using a 3D printed rectangular frame with dimensions of $3 \mathrm{~cm} \times 1.5 \mathrm{~cm}$, as shown in Figure 4A. The yarns were wrapped and fixed on the frame as a bundle (with 20 yarns). RSC were seeded on one end of yarn bundle at a density of $1 \times 10^{5}$ cells, and the cell migration and proliferation behaviors were continuously observed at predetermined time intervals throughout 14 days by using an MTT (Sigma) assay, as previously described. ${ }^{55}$

\subsection{Cell viability and proliferation characterization}

A Live/Dead assay (Invitrogen) was employed to characterize the cell viability and morphology

of cells cultured in different conditions, as previously described. ${ }^{56}$ A confocal laser scanning microscope (CLSM, LSM 710, Carl Zeiss) was utilized to obtain fluorescence images. The cell proliferation tests were conducted at predetermined time intervals by using an MTT assay.

\subsection{Immunofluorescent (IF) staining}

Cell-seeded samples were fixed in $4 \%$ paraformaldehyde, permeabilized in $0.2 \%$ Triton X-100, and then blocked with $1 \% \mathrm{BSA}$ overnight at $4{ }^{\circ} \mathrm{C} .{ }^{57}$ The samples were then treated with primary antibodies to S100 calcium-binding protein B (S100B, 1:250, Sigma), glial fibrillary acidic protein (GFAP, 1:200, Abcam), myelin basic proteins (MBP, 1:200, Abcam), and monoclonal anti- $\alpha$ smooth muscle actin ( $\alpha$ SMA)-Cy3 antibody (1:200, Sigma) overnight at $4{ }^{\circ} \mathrm{C}$. Secondary fluorescent antibodies were incubated for $2 \mathrm{~h}$, and nuclear counterstaining (Draq 5, 1: 1000, 
Thermo Scientific) was performed for 30 minutes at room temperature. The stained samples were imaged with a Zeiss 710 CLSM.

\subsection{Ribonucleic acid (RNA) isolation and Quantitative real time polymerase chain reaction} (qPCR)

Total RNA was extracted and purified through the sequential use of QIA-Shredder and RNeasy mini-kits (QIAgen) and was quantified by using NanodropTM OneC (Thermal Scientific). The extracted RNA was reverse transcribed into cDNA using an iScript cDNA synthesis kit (BioRad Laboratories). QPCR was performed using a StepOne ${ }^{\mathrm{TM}}$ Real-Time PCR System (Thermo Scientific) using SsoAdvanced SYBR Green Supermix (Bio-Rad). CDNA samples were analyzed for the housekeeping gene 18S rRNA and for the genes of interest, including S100B, GFAP, sex determining region Y-box 10 (SOX10), nerve growth factor receptor (NGFR), neural cell adhesion molecule 1 (NCAM1), fatty acid binding protein 7 (FABP7), MBP, myelin protein zero (MPZ), and myelin-associated glycoprotein (MAG). The relative expression level of each target gene was calculated using the comparative $\mathrm{Ct}\left(2^{-\Delta \Delta \mathrm{Ct}}\right)$ method and normalized to the level of $18 \mathrm{~S}$ rRNA. All detections were repeated three times independently. All primers used in this study are listed in

\section{Supplementary Table S1.}

\subsection{Statistical analysis}

All data is presented as the mean \pm standard deviation (SD) from three or more independent experiments. Pairwise comparisons between groups were conducted using ANOVA with Scheffé 
post-hoc tests in statistical analysis. Differences between groups were considered significant at $* * p<0.01$ and $* p<0.05$.

\section{RESULTS}

\subsection{Fabrication and morphologies of csNY}

A novel modified electrospinning device (Figure 1A) was developed to continuously coat the PLA MY (Figure 1B and D) with electrospun nanofibers, forming nanoyarns (NY) with core-sheath structures (Figure 1C and E-G). The microfiber core could thereby provide mechanical and structural stability, while the coated nanofiber sheath is expected to improve the biological properties. We fabricated three different csNY to demonstrate the feasibility of our csNY forming system (Figure 1E-G). They all employed PLA microfibers as the core layer, but utilized electrospun nanofibers made from PLGA, MeGel, or MeGel/PLGA mixtures as sheath layer (denoted as MeGel csNY, PLGA csNY or MeGel/PLGA csNY). Supplemental Figure S1 shows a clear PLA microfiber core and an electrospun MeGel/PLGA nanofiber sheath. A package of fabricated PLGA csNY (Figure 1C) demonstrated that csNY production was reproducible in a continuous and large-scale manner.

Figure 1D-G presented the surface morphologies of the raw PLA MY and three different types of csNY after electrospinning the nanofiber coating. The average diameters for PLA MY and the microfibers inside (Supplemental Figure S2) were 127.6 $\pm 6.6 \mu \mathrm{m}$ and 16.2 $\pm 0.6 \mu \mathrm{m}$, respectively.

Generally, for the three as-fabricated csNY, the PLA MY were evenly and completely covered by 
a layer of electrospun nanofibers without any PLA MY appearing on the csNY surface (Figure 1E-G). The nanofibrous coating increased the yarn diameter of the as-prepared csNY, and the mean yarn diameter was measured and calculated to be $130.9 \pm 5.5,132.1 \pm 6.2$, and $205.3 \pm 6.2 \mu \mathrm{m}$ for PLGA csNY, MeGel/PLGA csNY, and MeGel csNY, respectively (Supplemental Figure S2A). Most of the electropun nanofibers presented relatively smooth morphologies and were arranged well along the yarn in the longitudinal direction, for the PLGA csNY (Figure 1E) and MeGel/PLGA csNY (Figure 1F). However, the UV crosslinking and vacuum drying processes lead to poor fiber morphology retention on the surface of the MeGel csNY and notably increased the yarn diameter of the whole yarns, compared with the other two csNY (Figure 1G). This is probably due to the soft hydrogel nature of the pure $\mathrm{MeGel}$ component. The average diameters of the electrospun nanofibers in the sheath layer of the three different csNY were comparable (442.6 $\pm 278.1 \mathrm{~nm}$ for PLGA csNY vs. $468.0 \pm 220.0 \mathrm{~nm}$ for MeGel/PLGA csNY vs. 482.7.2 \pm 210.7 $\mathrm{nm}$ for MeGel csNY) (Supplemental Figure S2B), and all of them were significantly smaller than the PLA microfibers in the core layer. More than $60 \%$ of the coated nanofibers were highly aligned along the yarn's longitudinal direction in the PLGA csNY and MeGel/PLGA csNY (within $\pm 10^{\circ}$, Supplemental Figure S2C). FTIR spectra showed that a band at $1756 \mathrm{~cm}^{-1}$ was found in both PLGA csNY and MeGel/PLGA csNY, which presents the $\mathrm{C}=\mathrm{O}$ stretching vibration of ester carbonyl, and is the main chemical component for the PLGA polymer (Supplemental Figure S3). Moreover, a band at roughly $1647 \mathrm{~cm}^{-1}$ was observed in both MeGel csNY and MeGel/PLGA csNY, which is characteristic for the $-\mathrm{CONH}_{2}$ group exhibited in the MeGel polymer. FTIR results 
demonstrated that the MeGel/PLGA csNY were composed with both PLGA and MeGel components.

\subsection{Mechanical properties of csNY}

The representative load-elongation curves and calculated mechanical properties of the PLA MY and the three as-prepared csNY were presented in Figure 2. The results showed that when a tensile load was applied, all of the yarn samples exhibited a linear elastic region followed by a larger region of plastic deformation (Figure 2A). The tensile failure load of the PLGA csNY (277.7 \pm 9.0 $\mathrm{cN})$ and MeGel/PLGA csNY $(271.0 \pm 15.5 \mathrm{cN})$ were slightly higher than the PLA MY $(253.6 \pm 10.7$ $\mathrm{cN})$, but there was no significant change between the MeGel csNY $(259.0 \pm 14.0 \mathrm{cN})$ and PLA MY

(Figure 2B). Moreover, the coated PLGA nanofibers improved the Young's Modulus and ultimate strength of the PLGA csNY compared with the PLA MY, while no significant difference was found between the MeGel/PLGA csNY and PLA MY (Figure 2C and D). The coated MeGel nanofibers resulted in a significant decrease of the ultimate strength and Young's Modulus for MeGel csNY (Figure 2C and D). This is partially because of the significantly increased yarn diameter for the MeGel csNY. Comparable and similar failure strains, without significant differences, were found in all of the yarn materials (Figure 2E). The mechanical test results demonstrate that the inner PLA microfibers provide the structural integrity and mechanical properties for the obtained csNY. 

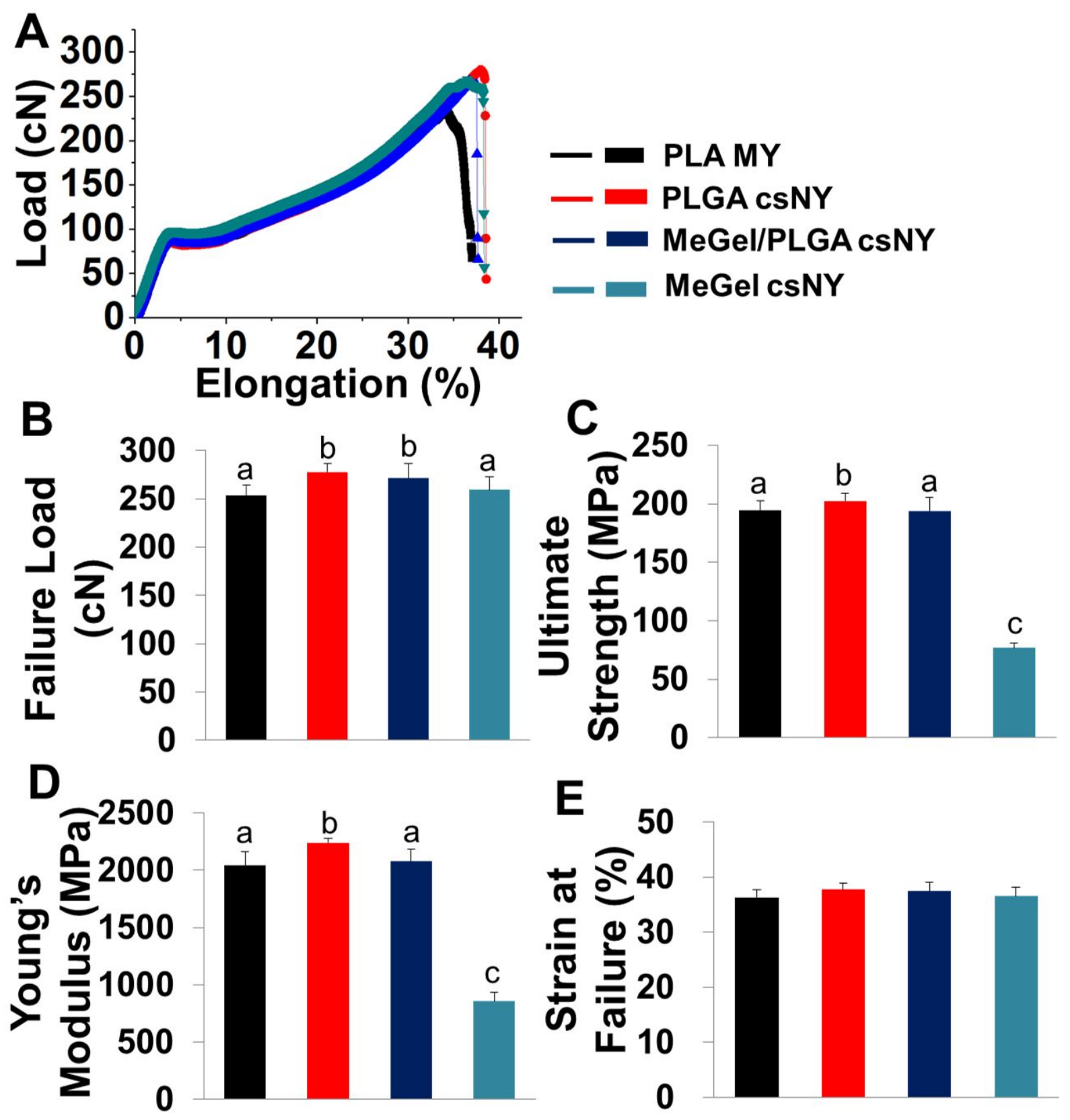

Figure 2. Characterization of tensile properties of the PLA MY and three different csNY. (A) Representative load-elongation curves. (B) Failure load (C) Ultimate tensile strength. (D) Young's modulus. (E) Strain at failure. ( $\mathrm{n}=15$; bars that do not share letters are significantly different from each other, $p<0.05)$. 


\subsection{Electrospun nanofiber coating promoted the adhesion, growth, and enhanced proliferation of hADMSC on csNY}

To further systematically investigate the effect of the electropun nanofiber coating on cell behavior, hADMSC were seeded on PLA MY and the three as-prepared csNY then cultured in GM for 14 days. HADMSC cultured on the four different yarn substrates showed high viability, with almost no dead cells being observed throughout 14 days of culture (Figure 3A-D). Interestingly, the cell adhesion characteristics of all of the csNY were superior to those of the PLA MY, and significantly more hADMSC were observed on all of the csNY compared to the PLA MY after 14-day culture. The MTT assay showed that the cells cultured on all of the csNY have significantly accelerated proliferation rates compared to the PLA MY throughout 14-day culture (Figure 3E). These results demonstrated that the coating of electrospun nanofibers could provide more adhesion sites for hADMSC, probably due to the significantly improved surface area-to-volume ratio, in comparison with traditional microfibers. Among the three different types of csNY, the MeGel csNY presented the highest proliferation rate. The reason for this could be that the coated MeGel contains more inherent cell adhesion domains compared to a synthetic polymer, like PLGA. 


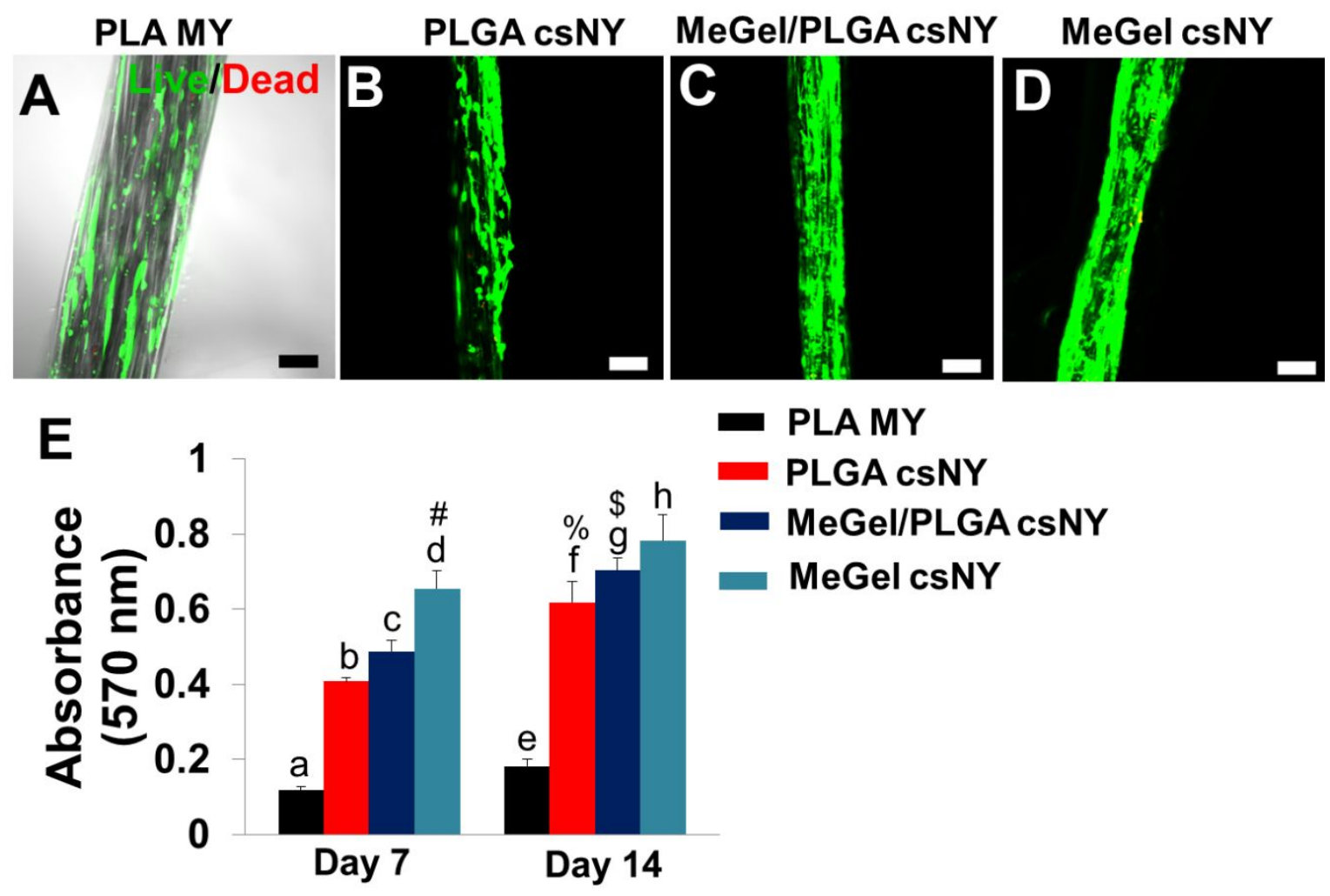

Figure 3. The coated electrospun nanofibers on the surface of csNY promoted hADMSC adhesion, growth, and proliferation. (A-D) Representative fluorescent images of the living (green) and dead (red) hADMSC seeded on PLA MY and three different types of csNY and conditioned in GM for 14 days. Scale bar $=100 \mu \mathrm{m}$. (C) Cell proliferation quantification by MTT assay at days 7 and 14 for hADMSC cultured on the different yarn materials in GM ( $\mathrm{n}=5$; bars that do not share letters are significantly different from each other $(p<0.05)$; there is no statistical difference between the groups with “\#” and "\%", and also the groups with “\#” and "\$”).

3.4 MeGel/PLGA csNY supported rSC migration, proliferation, and phenotype maintenance 
SC are the principal glial cells of the PN system, and they play a key role during the regeneration process of damaged PN. ${ }^{58}$ One of the essential requirements for PN grafts is to provide an instructive, structurally supportive microenvironment to support SC proliferation and migration. $\mathrm{MeGel} / \mathrm{PLGA} \operatorname{csNY}$ were selected to further test the migration and proliferation behaviors of $\mathrm{rSC}$ on csNY. We fixed a bundle of MeGel/PLGA csNY onto 3D printed frames and seeded rSC on one end of the yarn bundle. We then employed an MTT assay to observe the rSC migration and proliferation at predetermined time intervals throughout 14 days (Figure 4A). At day 1, the cells attached and concentrated on one end of the yarn bundle, and no significant cell migration was observed. After 14 days of cell culture, the rSC exhibited remarkable migration (about $2 \mathrm{~cm}$ ) along the long axis of the MeGel/PLGA csNY. Moreover, rSC proliferated continuously and possessed an obviously high proliferation rate from day 1 to day 14 on the MeGel/PLGA csNY (Figure 4B). IF staining showed that the $\mathrm{rSC}$ on the MeGel/PLGA csNY were positively active in producing SC-associated S100B protein after 14 days of culture (Figure 4C). These results confirmed that MeGel/PLGA csNY could provide a favorable surface and microenvironment for rSC attachment, migration, proliferation, and phenotype maintenance. 

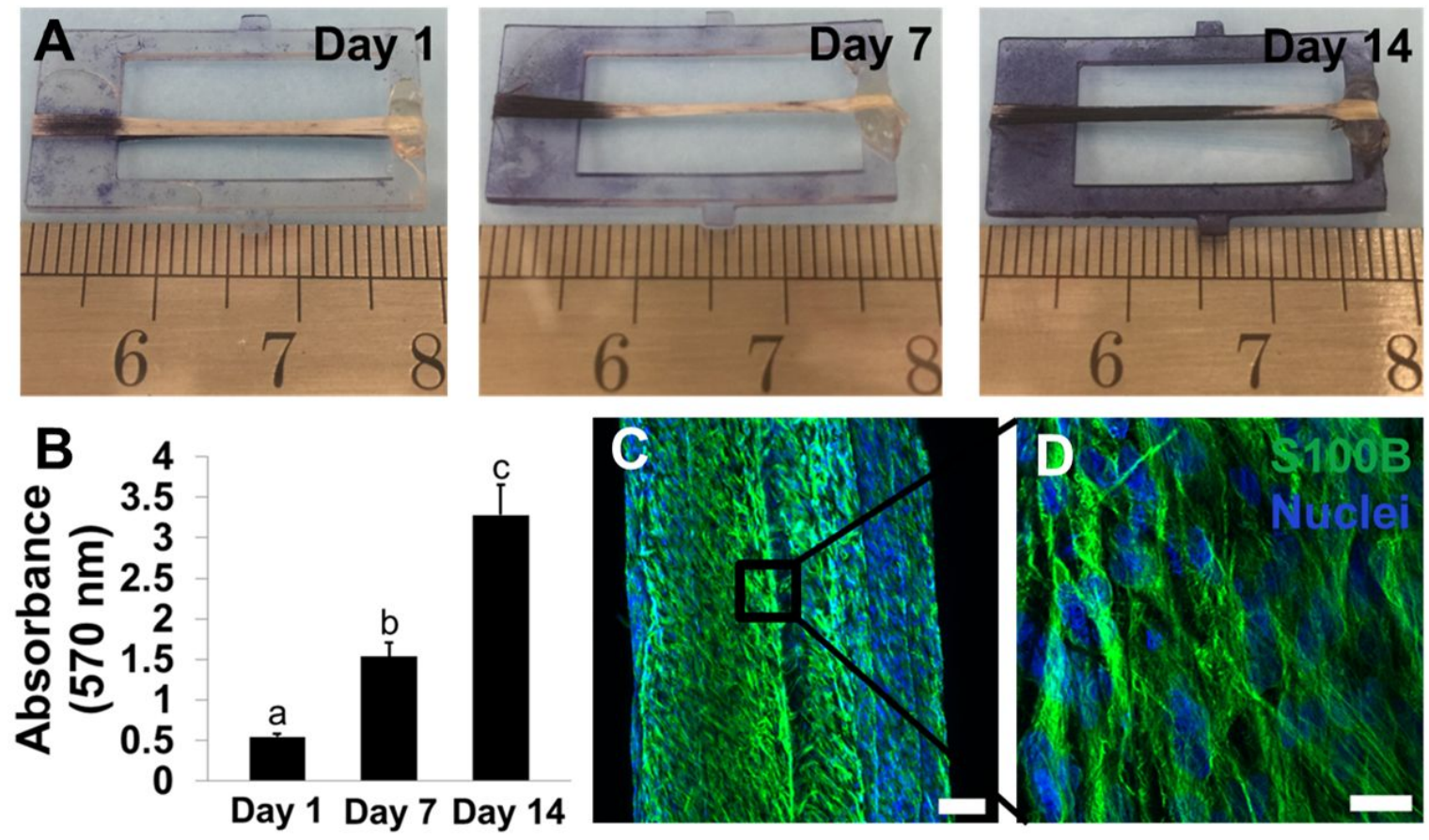

Figure 4. MeGel/PLGA csNY supported rSC migration, proliferation, and phenotype maintenance.

(A) The migration of rSC seeded on the MeGel/PLGA csNY bundles. (B) Cell proliferation quantification of rSC cultured on the MeGel/PLGA csNY bundles ( $\mathrm{n}=5$; bars that do not share letters are significantly different from each other, $p<0.01$ ). (C, D) IF staining for S100B (green) and nuclei (blue) for rSC on the MeGel/PLGA csNY bundles after 14 days of culture; Scale bars: $100 \mu \mathrm{m}$ for (C); $20 \mu \mathrm{m}$ for (D).

\subsection{MeGel/PLGA csNY promoted hADMSC differentiation into SC-like cells}

We next examined how the matrix topography and structure affected hADMSC growth and differentiation into hADMSC-SC. HADMSC were seeded and cultured on MeGel/PLGA csNY and 2D TCPS plates (as control). We chose MeGel/PLGA csNY due to their superior mechanical properties and support for cell proliferation. We found that the cell morphology varied in response 
to different substrate topographies. HADMSC on 2D TCPS plates exhibited a haphazard distribution (Figure 5A and B). In contrast, the nanofiber alignment on Me-Gel/PLGA csNY guided the expression of S100B and GFAP, two important positive markers of SC, along the arrangement direction of fibers. This demonstrated that hADMSC could sense the nanofibrous topographic features and regulate their morphology by the contact guidance phenomenon. We also found that hADMSC on the TCPS plates with GM presented a spindle-like shape with less positive expression of S100B and GFAP proteins after 14 days of culture. In comparison, after 14 days of induction, hADMSC on the TCPS plates with induction media (IM) showed a relatively narrow fusiform-like shape with a bipolar or tripolar structure, exhibited more SC-like morphologies, and presented an obviously enhanced S100B and GFAP expression. These indicated the potential differentiation capability of hADMSC into SC-like cells. Compared to the 2D TCPS plate, the cells cultured on MeGel/PLGA csNY, with both GM and IM, showed a high degree of S100B and a low degree of $\alpha$-smooth muscle actin ( $\alpha$ SMA, widely expressed in MSC) staining. 


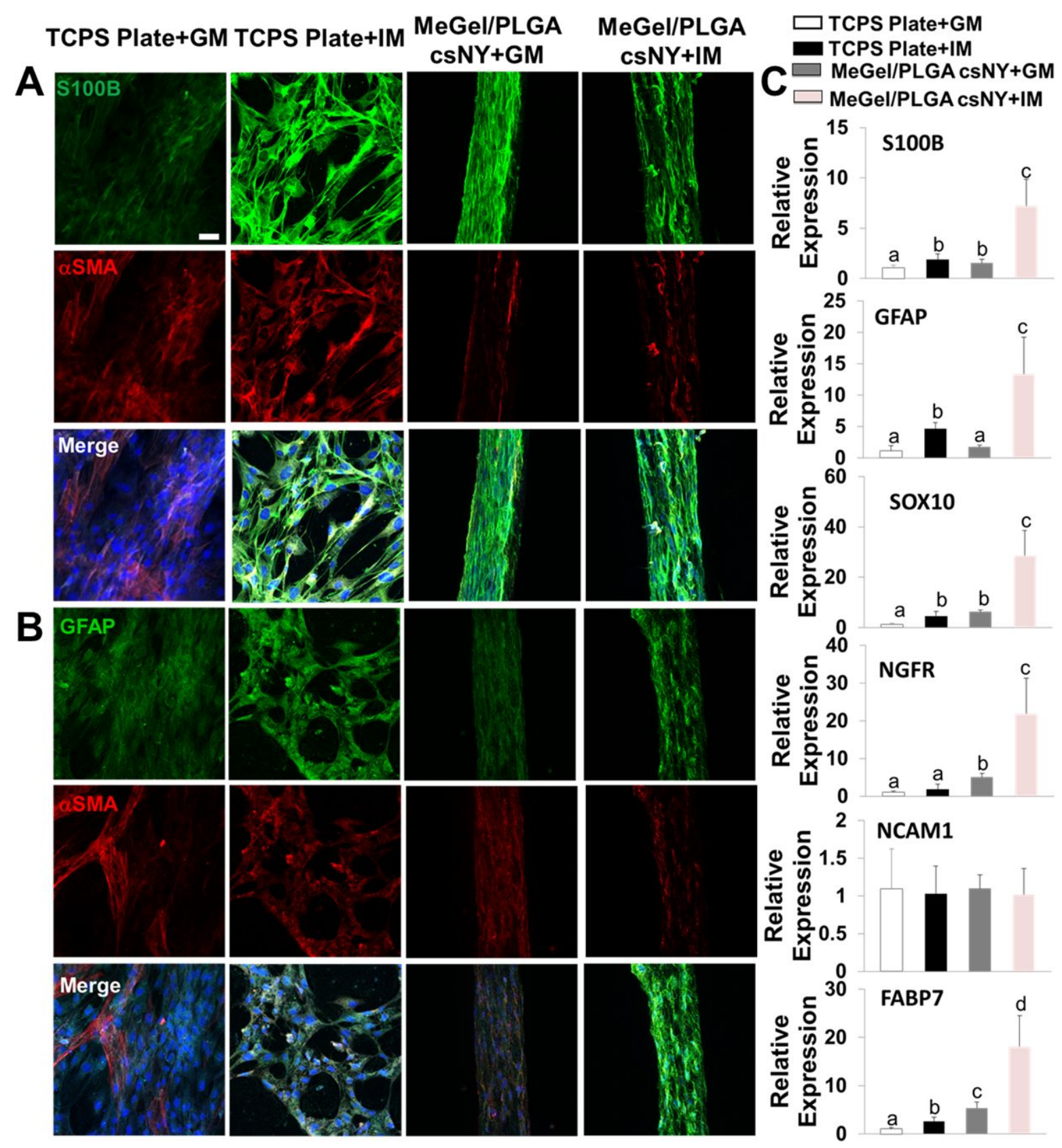

Figure 5. Me-Gel/PLGA csNY better enhanced hADMSC differentiation into SC-like cells, compared to 2D TCPS plates. HADMSC were seeded on MeGel/PLGA csNY and 2D TCPS plates and cultured in IM- I for 1 day, then in IM-II for 3 days, and finally in IM-II for 10 days. HADMSC seeded on MeGel/PLGA csNY and 2D TCPS plates were cultured in GM for 14 days, to serve as control groups. (A) IF staining for S100B (green), aSMA (red), and nuclei (blue). (B) 
IF staining for GFAP (green), $\alpha \mathrm{SMA}$ (red), and nuclei (blue). Scale bar $=50 \mu \mathrm{m}$. (C) qPCR analysis of S100B, GFAP, SOX10, NGFR, NCAM1, and FABP7 mRNA expression. Relative expression is presented as normalized to $18 \mathrm{~S}$ and expressed relative to HADMSC seeded on 2D TCPS plates and cultured in GM ( $\mathrm{n}=3$; bars that do not share letters are significantly different from each other, $p<0.05)$.

QPCR was employed to determine the relative expression levels of some early and immature SC markers in each group (Figure 5C). S100B is expressed by SC in most of the stages, and GFAP, SOX10, and NGFR are expressed by SC precursor or immature SC. Moreover, NCAM1 and FABP7 are related to early stage SC myelination. The analysis showed that hADMSC on the MeGel/PLGA csNY, with IM, presented significantly higher expression levels of S100B, GFAP, SOX10, NGFR, and FABP7, compared to those in the other three groups. Moreover, the relative expression levels of S100B, SOX10, and FABP7 of hADMSC on the MeGel/PLGA csNY, with GM, were also significantly higher than those on the TCPS plate with GM. Together, these results demonstrated that, compared to TCPS plate culture, MeGel/PLGA NY could provide an instructive microenvironment for hADSMC differentiation towards an SC-like phenotype by properly combining substrate morphology with medium induction. 

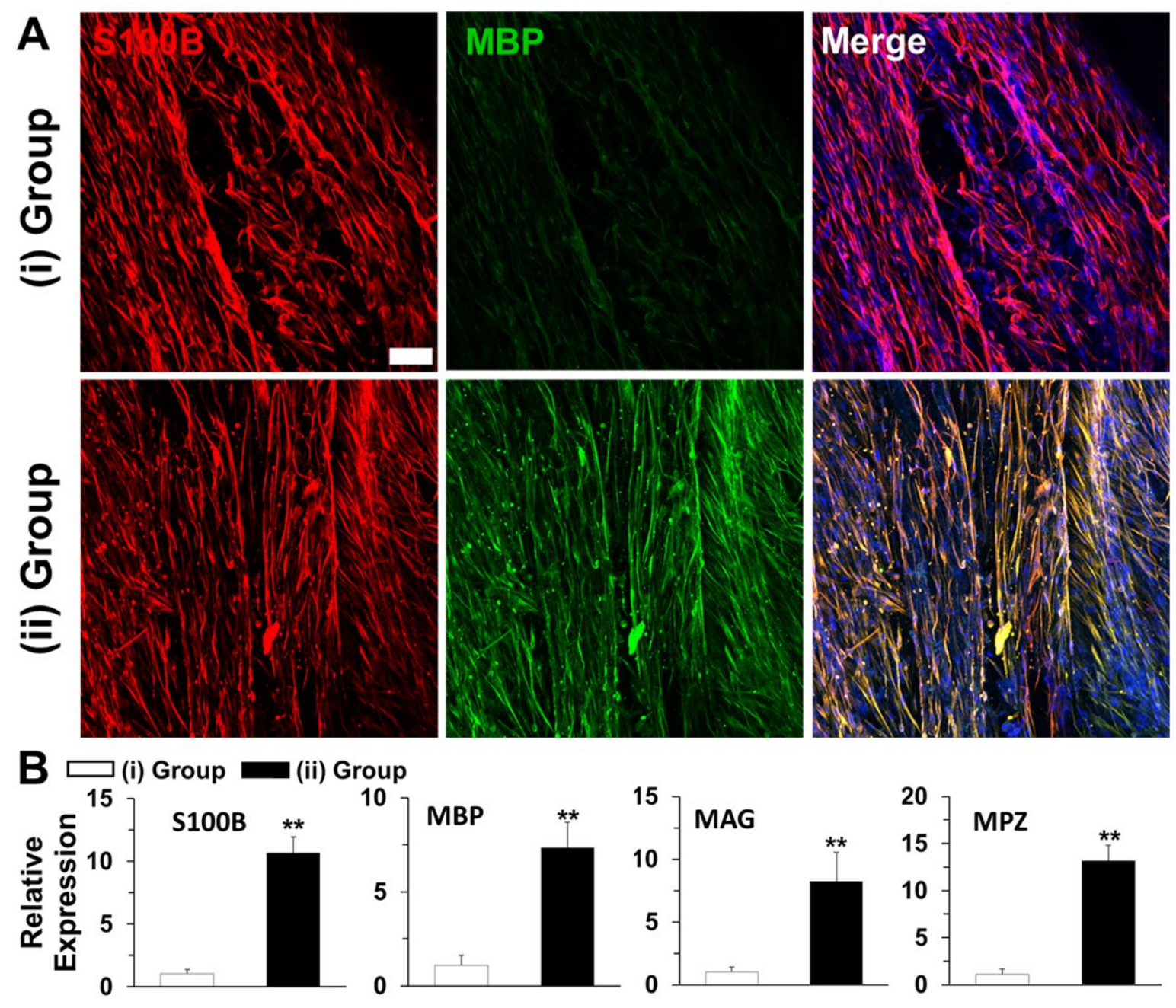

Figure 6. MeGel/PLGA csNY supported the myelination and maturation of hADMSC-SC under induction by neural differentiation medium. HADMSC were seeded on MeGel/PLGA csNY bundles and cultured in IM- I for 1 day, then in IM-II for 3 days, and finally in IM-II for 10 days. After that, these obtained hADMSC-SC-seeded csNY bundles were divided into two groups: (i) maintained in IM-III for another 14 days; (ii) utilized IM-IV (neural differentiation medium) for further induction for 14 days. (A) IF staining for MBP (green), GFAP (red), and nuclei (blue). Scale bar $=100 \mu \mathrm{m}$. (B) qPCR analysis of S100B, MBP, MPZ, and MAG mRNA expression. 
Relative expression is presented as normalized to $18 \mathrm{~S}$ and expressed relative to group (i) ( $\mathrm{n}=3$; $\left.{ }^{* *} p<0.01\right)$.

\subsection{Appropriate medium induction enhanced the differentiation of hADMSC into} myelinating SC-like cells

Remyelination of axons by SC is critical for the successful treatment of damaged PN defects. Unfortunately, the methods to direct and enhance hADMSC differentiation into myelinating SClike cells remain limited. The MeGel/PLGA csNY have been proven above to possess an excellent capacity to regulate hADMSC differentiation into SC-like cells by enhancing the expressions of some early and immature SC markers, including GFAP and S100B (Figure 5A and B). However, no obvious expression of myelin basic protein (MBP, a marker for mature SC) was observed for hADMSC cultured on the MeGel/PLGA csNY after 10 days of induction in IM-III (Supplemental Fig. S4). However, it is significantly necessary to promote the SC-like cell maturation and myelination. After the induction of hADMSC into hADMSC-SC, we further utilized neural progenitor medium to treat hADMSC-SC on MeGel/PLGA csNY for another 14 days. IF staining showed that comparably robust S100B expression was found in both groups, with and without neural differentiation medium (Figure 6A). However, almost no MBP protein expression was detected in the group without neural progenitor medium treatment, whereas hADMSC-SC, after further induction in neural progenitor medium, expressed robust MBP. The qPCR results 
confirmed that induction with neural progenitor medium significantly upregulated the SC myelination-related gene expressions, including MBP, MAG, and MPZ (Figure 6B).

\section{DISCUSSION}

Native PN, consisting of motor and sensory axons and supportive tissues, exhibit a multiple hierarchical architecture. ${ }^{58,59}$ Motor or sensory axons from group A and group B nerve fibers are surrounded by myelinated SC sheaths and bundled together to form fascicles. A group of aligned fascicles is then well-organized into an anatomically defined nerve trunk. Therefore, when a biomimetic scaffold is designed for PN regeneration, it is essentially important to consider the high complexity of the natural PN structure. Meanwhile, previous experimental and clinical studies have revealed that hollow NGC alone cannot perform satisfactorily, especially for large PN defects. ${ }^{11}$ Hollow NGC were assuredly beneficial for avoiding scar tissue formation and concentrating the regenerative microenvironment, but they failed to provide native PN-like inner microenvironments for guiding cell migration into the tube and promoting further functional regeneration. Therefore, employing micro-structured NGC-infilling biomaterials (such as fibers, hydrogels, and sponges) as topological cues represent promising candidates to enhance PN regeneration..$^{60,61}$

Fibers have been recognized as a very attractive infilling substrate to bio-mimic the architecture of axons in the PN system. ${ }^{62}$ Some studies employed wet or dry spinning to fabricate microfibers with diameters of $>10 \mu \mathrm{m}$. They also demonstrated that adding microfibers to NGC could provide 
inner contact guidance and increase nutrient and waste permeability, which could enhance the overall cross-sectional area of regenerated nerve tissues in comparison with hollow NGC without microfibers. ${ }^{63,64}$ Recently, with the development of nanofabrication techniques, electrospinning has been recognized as a simple and straightforward method to fabricate nanofibers with diameters in the range of 500-1000 nm. ${ }^{65-67}$ Some studies indicated that electrospun nanofibers could closely mimic the diameter scale and topographical cues of the axons of native PN, which could significantly promote cell adhesion, spreading, proliferation, as well as SC-specific differentiation of stem cells. ${ }^{68,69}$ Moreover, advances in hydrogel technologies have spurred the development of hydrogel fibers. ${ }^{70,71}$ Some hydrogel precursors, such as collagen, gelatin, and fibronectin, which could better mimic the physiological and biomechanical properties of native tissues, have been fabricated into fibers and were found to promote the survival and neurite outgrowth of nerve cells and provided a consistent promoting effect on the regeneration of injured nerves. ${ }^{23,62}$ Note that some researchers also incorporated various growth factors, and even living cells, into/onto fibers to endow the modified fiber substrates with both contact-guidance and biological cues, which provided a synergetic function for damaged PN regeneration. ${ }^{72-75}$ In our current study, we developed a modified electrospinning device to continuously coat biodegradable PLA MY with conformal layers of electrospun nanofibers. By utilizing this method, we successfully generated nano-surface-possessed and mechanically-strong core-sheath csNY consisting of a microfiber core and nanofiber sheath, which could combine the advantages of both nanofibers and microfibers to better resemble the aligned fibrous architectures of axons and fascicles at the microscale level. 
Moreover, multiple csNY can be further employed to form bundles to replicate the hierarchical architecture of a PN trunk at the macroscale level.

Biocompatibility and biomechanical performance are two major concerns for an ideal structurally supportive PN graft. ${ }^{76}$ These required properties are mainly dependent on the graft material and structure. In this study, we fabricated three different csNY, i.e. PLGA csNY, MeGel/PLGA csNY, and MeGel csNY, with PLA MY as core layer. Live/dead and MTT assays demonstrated that the coated electrospun nanofibers on PLA MY provided more cell adhesion sites and significantly improved the attachment and proliferation of HADMSC, compared to PLA MY alone. The longitudinally oriented nanofibrous surface topography was a dominating factor in positively affecting the interactions between cells and biomaterials. We also found that the nanofibers coated with MeGel could better mimic not only the nanofibrous scale and structure but also the physiochemical properties of native ECM. As supported by prior studies, our findings confirmed that the combination of reduced fiber scale and appropriate material components could offer unique biomaterial surface properties, such as a high surface area-to-volume ratio, which is favorable for cell adhesion and growth, and topographical signals that are favorable for directing cellular functions. ${ }^{77,78}$

Fiber-based intraluminal filling material is required to possess appropriate mechanical properties, which could better maintain its shape in order to bridge the gap between the proximal and distal nerve ends during and after implantation. ${ }^{21}$ Our results demonstrated that all of the csNY fabricated in this study have excellent structural integrity and mechanical properties. We compared the shape 
retention of a bundle of MeGel/PLGA csNY with a bundle of electrospun nanofibrous yarns consisting of pure PLGA nanofibers and found that the shape retention was obviously enhanced in MeGel/PLGA csNY group (Supplemental Figure S5). Previous studies have demonstrated that electrospun nanofibers were too flexible to maintain the aligned structure without kinking after implantation, which was not beneficial to support SC ingrowth and axonal regeneration. ${ }^{22,79}$ Therefore, our csNY should have a suitable stiffness for bridging the gap without bending, but in vivo implantation is required for further verification.

SC play a critical role during repair and regeneration of an injured PN system. Following injuries, if the nerve gap is very short, a fibrin cable will form spontaneously between the two nerve stumps. Then the proliferated SC will migrate along with the fibrin cable into the gap, forming a biological tissue cable, known as bands of Büngner, for directing subsequent axonal regrowth from the proximal end to the distal end at a rate of up to $1 \mathrm{~mm}$ per day. ${ }^{80} \mathrm{In}$ the case of large nerve gaps, which lack the formation of aligned fibrin bridge, there was limited migration of native SC into the lesion site, inevitably resulting in reduced cellular activity and an inferior regeneration outcome. ${ }^{23}$ Therefore, engineering a topography that closely mimics a native fibrin cable is one of the major criteria for NGC-infilling materials. In the present study, we tested the cell-matrix interaction between $\mathrm{rSC}$ and $\mathrm{MeGel} / \mathrm{PLGA}$ csNY. Aligned MeGel/PLGA nanofibers on the surface of MeGel/PLGA csNY directed linear alignment and migration of rSC. Directionally targeted alignment and migration did not occur in 2D TCPS plates. ${ }^{81,82}$ In addition, the high surface area-to-volume ratio provided by MeGel/PLGA nanofibers supported rSC adhesion and growth, 
as well as phenotype maintenance. Our csNY may mimic the fibrin cable and may be utilized to model the formation of bands of Büngner in vitro.

The combinatorial use of stem cells and fibrous materials has become an important approach in PN tissue engineering. Recently several studies have explored the differentiation of stem cells from different sources towards SC-like cells based on 2D TCPS plate tests. ${ }^{83,84}$ However, the lack of effective mimicking of the native tissue microenvironment in $2 \mathrm{D}$ substrates skews the differentiation behavior. ${ }^{85}$ Previous studies also demonstrated that an aligned nanofibrous structure could promote cell attachment, proliferation, and differentiation of stem cells. ${ }^{86,87}$ In this study, the differentiation of hADMSC was conducted directly on our developed MeGel/PLGA csNY. Our results demonstrated that an aligned fibrous surface topography significantly promoted the differentiation of hADMSC into SC-like cells. Moreover, we also found that neural differentiation media could significantly promote further differentiation of hADMSC-SC into myelinating SClike cells with a higher expression of the myelination-associated genes (MBP, MAG, and MPZ) on MeGel/PLGA csNY. At the current stage, the mechanism of induction by neural differentiation medium is unclear, and future studies should be conducted to better control the differentiation. In response to axonal interruption and degeneration, $\mathrm{SC}$ are activated and become non-myelinating cells with an upregulated expression of cytokines in order to recruit macrophages and with a downregulated expression of myelin-associated genes. ${ }^{88}$ The activation and de-differentiation stages are transient, and after injury, SC undergo re-differentiation and transform back into myelinating cells to promote nerve regeneration. ${ }^{89} \mathrm{It}$ is unclear which state of hADMSC-SC should 
be used for implantation and has better therapeutic effects on guiding the axons from the proximal stump across the gap. Our current study demonstrated that we can control the hADMSC differentiation stage on our csNY and provide a different choice for further in vivo implantation.

In summary, our csNY has four major advantages for PN engineering: (1) imitation of the hierarchically and anisotropically aligned fiber structure of axons and fascicles, (2) maintaining adequate mechanical support for the regenerating tissues, (3) recreation of essential topographical and physiochemical features for attachment, proliferation, alignment, and hADMSC-SC differentiation, and (4) mimicking the fibrin cable to promote the formation of bands of Büngner for directing subsequent axonal regrowth. Overall, it is envisioned that our csNY could be effectively utilized to serve not only as in vitro culture models for understanding SC-axon interaction but also as promising NGC-infilling materials for PN regeneration applications. Future studies should further elucidate the signaling pathway during hADMSC differentiation towards myelinating hADMSC-SC and the roles that csNY play during the differentiation process. Signaling pathways like Raf/ERK and c-Jun are probably involved, due to their roles in mediating the repair and myelination phenotypes of SC in vivo ${ }^{80}$ Furthermore, the in vivo validation of these csNY-based infilling substrates with hADMSC-SC will be required.

\section{CONCLUSIONS}

In this study, we developed a novel approach for fabricating csNY with a PLA MY core and nanofibrous coatings by modifying a typical electrospinning setup. The aligned nanofibrous 
topography endowed the obtained csNY with both the ability of physical contact-guidance and bioactive chemical cues to facilitate hADMSC and $\mathrm{rSC}$ attachment, proliferation, and directional alignment and migration. Meanwhile, the inherent PLA microfibers in the csNY core layer provided a robust mechanical performance for the structural integrity and load resistance for PN repair. Our results demonstrated that MeGel/PLGA csNY promoted hADMSC differentiation into hADMSC-SC better than 2D TCPS plates, and they supported further differentiation into myelinating hADSMC-SC, under the appropriate induction medium. This study therefore provides fresh insights into the regulation of stem cell differentiation into myelinating SC-like phenotypes and the fabrication of native PN ECM-like biological intraluminal filling substitutes for PN regeneration.

\section{ASSOCIATED CONTENT}

\section{Supporting Information}

The Supporting Information is available on the website.

\section{AUTHOR INFORMATION}

\section{Corresponding Author}

*E-mail: bin.duan@unmc.edu; Tel: +1 4025599637

\section{Notes}

The authors declare no competing financial interest. 


\section{ACKNOWLEDGMENTS}

This work has been supported by Mary \& Dick Holland Regenerative Medicine Program start-up grant, Nebraska Research Initiative funding, National Institutes of Health (R01 AR073225), and Nebraska Stem Cell Research Project Grant. Support for the UNMC Advanced Microscopy Core Facility was provided by the Nebraska Research Initiative, the Fred and Pamela Buffett Cancer Center Support Grant (P30CA036727), and an Institutional Development Award (IDeA) from the NIGMS of the NIH (P30GM106397).

\section{ABBREVIATION}

NGC, Nerve guidance conduits; PN, peripheral nerves; csNY, core-sheath nanoyarns; hADMSC, human adipose derived mesenchymal stem cells; SC, Schwann cells; TCPS plate, tissue culture polystyrene plate; rSC, rabbit Schwann cells; Gel, Gelatin; MeGel, methacrylated gelatin; PLGA, poly (lactic-co-glycolic) acid; NMD, rotating neutral metal disc; NHMR, static neutral hollow metal rod; GM, growth medium; IM, induction media; DMEM/F12, Dulbecco's modified Eagle's medium/F12; FBS, fetal bovine serum; P/S, penicillin/streptomycin; PBS, phosphate buffered saline; SEM, scanning electron microscope; IF, Immunofluorescent; PLA, polylactic acid; MY, microfiber yarns; bFGF, basic fibroblast growth factor; PDGF-AA, platelet derived growth factorAA; RNA, Ribonucleic acid; QPCR, Quantitative real time polymerase chain reaction; S100B, S100 calcium-binding protein B; GFAP, glial fibrillary acidic protein; MBP, myelin basic proteins; 
aSMA, $\alpha$-smooth muscle actin; NGFR, nerve growth factor receptor; NCAM1, neural cell adhesion molecule 1; FABP7, fatty acid binding protein 7; MPZ, myelin protein zero; MAG, myelin-associated glycoprotein.

\section{REFERENCES}

(1) Faroni, A.; Mobasseri, S. A.; Kingham, P. J.; Reid, A. J., Peripheral nerve regeneration: experimental strategies and future perspectives. Adv. Drug Deliv. Rev. 2015, 82, 160-167, DOI: 10.1016/j.addr.2014.11.010.

(2) Scheib, J.; Höke, A., Advances in peripheral nerve regeneration. Nat. Rev. Neurol. 2013, 9 (12), 668, DOI: 10.1038/nrneurol.2013.227.

(3) Chiono, V.; Tonda-Turo, C., Trends in the design of nerve guidance channels in peripheral nerve tissue engineering. Prog. Neurobiol. 2015, 131, 87-104, DOI: 10.1016/j.pneurobio.2015.06.001.

(4) Haring, A. P.; Sontheimer, H.; Johnson, B. N., Microphysiological human brain and neural systems-on-a-Chip: potential alternatives to small animal models and emerging platforms for drug discovery and personalized medicine. Stem Cell Rev. Rep. 2017, 13 (3), 381-406, DOI: 10.1007/s12015-017-9738-0.

(5) Grinsell, D.; Keating, C., Peripheral nerve reconstruction after injury: a review of clinical and experimental therapies. BioMed Res. Int. 2014, 2014, 698256, DOI: 10.1155/2014/698256.

(6) Li, R.; Liu, Z.; Pan, Y.; Chen, L.; Zhang, Z.; Lu, L., Peripheral nerve injuries treatment: a systematic review. Cell Biochem. Biophys. 2014, 68 (3), 449-454, DOI: 10.1007/s12013-0139742-1.

(7) Gaudin, R.; Knipfer, C.; Henningsen, A.; Smeets, R.; Heiland, M.; Hadlock, T., Approaches to peripheral nerve repair: generations of biomaterial conduits yielding to replacing autologous nerve grafts in craniomaxillofacial surgery. BioMed Res. Int. 2016, 2016, 3856262, DOI: $10.1155 / 2016 / 3856262$.

(8) Zhu, W.; Tringale, K. R.; Woller, S. A.; You, S.; Johnson, S.; Shen, H.; Schimelman, J.; Whitney, M.; Steinauer, J.; Xu, W., Rapid continuous 3D printing of customizable peripheral nerve guidance conduits. Mater. Today 2018, 21, 951, DOI: 10.1016/j.mattod.2018.04.001.

(9) Naryan, S. K.; Arumugam, M.; Chittoria, R., Outcome of human peripheral nerve repair interventions using conduits: a systematic review. J. Neurol. Sci. 2018, 396, 18, DOI: 10.1016/j.jns.2018.10.012. 
(10) Lin, T.; Liu, S.; Chen, S.; Qiu, S.; Rao, Z.; Liu, J.; Zhu, S.; Yan, L.; Mao, H.; Zhu, Q., Hydrogel derived from porcine decellularized nerve tissue as a promising biomaterial for repairing peripheral nerve defects. Acta Biomater. 2018, 73, 326-338, DOI: 10.1016/j.actbio.2018.04.001. (11) Sarker, M.; Naghieh, S.; McInnes, A. D.; Schreyer, D. J.; Chen, X., Strategic Design and Fabrication of Nerve Guidance Conduits for Peripheral Nerve Regeneration. Biotechnol. J. 2018, 13, 1700635, DOI: 10.1002/biot.201700635.

(12) Li, B.; Qiu, T.; Iyer, K. S.; Yan, Q.; Yin, Y.; Xie, L.; Wang, X.; Li, S., PRGD/PDLLA conduit potentiates rat sciatic nerve regeneration and the underlying molecular mechanism. Biomaterials 2015, 55, 44-53, DOI: 10.1016/j.biomaterials.2015.03.028.

(13) Zhang, W.; Zhang, L.; Liu, J.; Zhang, L.; Zhang, J.; Tang, P., Repairing sciatic nerve injury with an EPO-loaded nerve conduit and sandwiched-in strategy of transplanting mesenchymal stem cells. Biomaterials 2017, 142, 90-100, DOI: 10.1016/j.biomaterials.2017.06.024.

(14) Bozkurt, A.; Boecker, A.; Tank, J.; Altinova, H.; Deumens, R.; Dabhi, C.; Tolba, R.; Weis, J.; Brook, G.; Pallua, N., Efficient bridging of $20 \mathrm{~mm}$ rat sciatic nerve lesions with a longitudinally micro-structured collagen scaffold. Biomaterials 2016, 75, 112-122, DOI: 10.1016/j.biomaterials.2015.10.009.

(15) Park, S. Y.; Ki, C. S.; Park, Y. H.; Lee, K. G.; Kang, S. W.; Kweon, H. Y.; Kim, H. J., Functional recovery guided by an electrospun silk fibroin conduit after sciatic nerve injury in rats. J. Tissue Eng. Regen. Med. 2015, 9 (1), 66-76, DOI: 10.1002/term.1615.

(16) Suo, H.; Wang, Z.; Dai, G.; Fu, J.; Yin, J.; Chang, L., Polyacrylonitrile Nerve Conduits With Inner Longitudinal Grooved Textures to Enhance Neuron Directional Outgrowth. $J$. Microelectromech. Syst. 2018, 27 (3), 457-463, DOI: 10.1109/JMEMS.2018.2810097.

(17) Mobasseri, A.; Faroni, A.; Minogue, B. M.; Downes, S.; Terenghi, G.; Reid, A. J., Polymer scaffolds with preferential parallel grooves enhance nerve regeneration. Tissue Eng. Part A 2015, 21 (5-6), 1152-1162, DOI: 10.1089/ten.tea.2014.0266.

(18) Liu, S.; Sun, X.; Wang, T.; Chen, S.; Zeng, C. G.; Xie, G.; Zhu, Q.; Liu, X.; Quan, D., Nanofibrous and ladder-like multi-channel nerve conduits: Degradation and modification by gelatin. Mater. Sci. Eng. C-Mater. Biol. Appl. 2018, 83, 130-142, DOI: 10.1016/j.msec.2017.11.020.

(19) Shah, M. B.; Chang, W.; Zhou, G.; Glavy, J. S.; Cattabiani, T. M.; Yu, X., Novel spiral structured nerve guidance conduits with multichannels and inner longitudinally aligned nanofibers for peripheral nerve regeneration. Journal of Biomedical Materials Research Part B: Applied Biomaterials 2019, 107B, 1410-1419, DOI: 10.1002/jbm.b.34233.

(20) Sabongi, R. G.; Fernandes, M.; dos Santos, J. B. G., Peripheral nerve regeneration with conduits: use of vein tubes. Neural. Regen. Res. 2015, 10 (4), 529-533, DOI: 10.4103/16735374.155428.

(21) Muheremu, A.; Ao, Q., Past, present, and future of nerve conduits in the treatment of peripheral nerve injury. BioMed Res. Int. 2015, 2015, 237507, DOI: 10.1155/2015/237507. 
(22) Jing, W.; Ao, Q.; Wang, L.; Huang, Z.; Cai, Q.; Chen, G.; Yang, X.; Zhong, W., Constructing conductive conduit with conductive fibrous infilling for peripheral nerve regeneration. Chem. Eng. J. 2018, 345, 566-577, DOI: 10.1016/j.cej.2018.04.044.

(23) Du, J.; Liu, J.; Yao, S.; Mao, H.; Peng, J.; Sun, X.; Cao, Z.; Yang, Y.; Xiao, B.; Wang, Y., Prompt peripheral nerve regeneration induced by a hierarchically aligned fibrin nanofiber hydrogel. Acta biomater. 2017, 55, 296-309, DOI: 10.1016/j.actbio.2017.04.010.

(24) Peng, S. W.; Li, C. W.; Chiu, M.; Wang, G. J., Nerve guidance conduit with a hybrid structure of a PLGA microfibrous bundle wrapped in a micro/nanostructured membrane. Int. J. Nanomedicine 2017, 12, 421, DOI: 10.2147/IJN.S122017.

(25) Li, D.; Pan, X.; Sun, B.; Wu, T.; Chen, W.; Huang, C.; Ke, Q.; EI-Hamshary, H. A.; AlDeyab, S. S.; Mo, X., Nerve conduits constructed by electrospun P (LLA-CL) nanofibers and PLLA nanofiber yarns. J. Mater. Chem. B 2015, 3 (45), 8823-8831, DOI: 10.1039/C5TB01402F. (26) Wang, Y.; Wang, W.; Wo, Y.; Gui, T.; Zhu, H.; Mo, X.; Chen, C. C.; Li, Q.; Ding, W., Orientated guidance of peripheral nerve regeneration using conduits with a microtube array sheet (MTAS). ACS Appl. Mater. Interfaces 2015, 7 (16), 8437-8450, DOI: 10.1021/acsami.5b00215.

(27) Pedde, R. D.; Mirani, B.; Navaei, A.; Styan, T.; Wong, S.; Mehrali, M.; Thakur, A.; Mohtaram, N. K.; Bayati, A.; Dolatshahi - Pirouz, A., Emerging biofabrication strategies for engineering complex tissue constructs. Adv. Mater. 2017, 29 (19), 1606061, DOI: 10.1002/adma.201606061. (28) Sun, Y.; Li, W.; Wu, X.; Zhang, N.; Zhang, Y.; Ouyang, S.; Song, X.; Fang, X.; Seeram, R.; Xue, W., Functional self-assembling peptide nanofiber hydrogels designed for nerve degeneration. ACS Appl. Mater. Interfaces 2016, 8 (3), 2348-2359, DOI: 10.1021/acsami.5b11473.

(29) Li, A.; Hokugo, A.; Yalom, A.; Berns, E. J.; Stephanopoulos, N.; McClendon, M. T.; Segovia, L. A.; Spigelman, I.; Stupp, S. I.; Jarrahy, R., A bioengineered peripheral nerve construct using aligned peptide amphiphile nanofibers. Biomaterials 2014, 35 (31), 8780-8790, DOI: 10.1016/j.biomaterials.2014.06.049.

(30) Shakhbazau, A.; Kawasoe, J.; Hoyng, S. A.; Kumar, R.; van Minnen, J.; Verhaagen, J.; Midha, R., Early regenerative effects of NGF-transduced Schwann cells in peripheral nerve repair. Mol Cell Neurosci 2012, 50 (1), 103-112, DOI: 10.1016/j.mcn.2012.04.004.

(31) Webber, C.; Zochodne, D., The nerve regenerative microenvironment: early behavior and partnership of axons and Schwann cells. Exp. Neurol. 2010, 223 (1), 51-59, DOI: 10.1016/j.expneurol.2009.05.037.

(32) Sullivan, R.; Dailey, T.; Duncan, K.; Abel, N.; Borlongan, C. V., Peripheral nerve injury: stem cell therapy and peripheral nerve transfer. Int. J. Mol. Sci. 2016, 17 (12), 2101, DOI: 10.3390/ijms17122101.

(33) Fairbairn, N. G.; Meppelink, A. M.; Ng-Glazier, J.; Randolph, M. A.; Winograd, J. M., Augmenting peripheral nerve regeneration using stem cells: A review of current opinion. World J. Stem Cells 2015, 7 (1), 11-26, DOI: 10.4252/wjsc.v7.i1.11. 
(34) Jenkins, P. M.; Laughter, M. R.; Lee, D. J.; Lee, Y. M.; Freed, C. R.; Park, D., A nerve guidance conduit with topographical and biochemical cues: potential application using human neural stem cells. Nanoscale Res. Lett. 2015, 10 (1), 264, DOI: 10.1186/s11671-015-0972-6.

(35) Weber, M.; Apostolova, G.; Widera, D.; Mittelbronn, M.; Dechant, G.; Kaltschmidt, B.; Rohrer, H., Alternative Generation of CNS Neural Stem Cells and PNS Derivatives from Neural Crest-Derived Peripheral Stem Cells. Stem Cells 2015, 33 (2), 574-588, DOI: 10.1002/stem.1880. (36) Sparling, J. S.; Bretzner, F.; Biernaskie, J.; Assinck, P.; Jiang, Y.; Arisato, H.; Plunet, W. T.; Borisoff, J.; Liu, J.; Miller, F. D., Schwann cells generated from neonatal skin-derived precursors or neonatal peripheral nerve improve functional recovery after acute transplantation into the partially injured cervical spinal cord of the rat. J. Neurosci. 2015, 35 (17), 6714-6730, DOI: 10.1523/JNEUROSCI.1070-14.2015.

(37) Stratton, J. A.; Shah, P. T.; Kumar, R.; Stykel, M. G.; Shapira, Y.; Grochmal, J.; Guo, G. F.; Biernaskie, J.; Midha, R., The immunomodulatory properties of adult skin-derived precursor S chwann cells: implications for peripheral nerve injury therapy. Eur. J. Neurosci. 2016, 43 (3), 365375, DOI: 10.1111/ejn.13006.

(38) Guo, Z. Y.; Sun, X.; Xu, X. L.; Zhao, Q.; Peng, J.; Wang, Y., Human umbilical cord mesenchymal stem cells promote peripheral nerve repair via paracrine mechanisms. Neural Regen. Res. 2015, 10 (4), 651-658, DOI: 10.4103/1673-5374.155442.

(39) Nagamura-Inoue, T.; He, H., Umbilical cord-derived mesenchymal stem cells: their advantages and potential clinical utility. World J. Stem Cells 2014, 6 (2), 195-202, DOI: 10.4252/wjsc.v6.i2.195.

(40) Yousefifard, M.; Nasirinezhad, F.; Manaheji, H. S.; Janzadeh, A.; Hosseini, M.; Keshavarz, M., Human bone marrow-derived and umbilical cord-derived mesenchymal stem cells for alleviating neuropathic pain in a spinal cord injury model. Stem Cell Res. Ther. 2016, 7 (1), 36, DOI: $10.1186 / \mathrm{s} 13287-016-0295-2$.

(41) Schäfer, S.; Berger, J. V.; Deumens, R.; Goursaud, S.; Hanisch, U.-K.; Hermans, E., Influence of intrathecal delivery of bone marrow-derived mesenchymal stem cells on spinal inflammation and pain hypersensitivity in a rat model of peripheral nerve injury. J. Neuroinflammation 2014, 11 (1), 157, DOI: 10.1186/s12974-014-0157-8.

(42) Zack-Williams, S. D.; Butler, P. E.; Kalaskar, D. M., Current progress in use of adipose derived stem cells in peripheral nerve regeneration. World J. Stem Cells 2015, 7 (1), 51, DOI: 10.4252/wjsc.v7.i1.51.

(43) Sowa, Y.; Kishida, T.; Imura, T.; Numajiri, T.; Nishino, K.; Tabata, Y.; Mazda, O., Adiposederived stem cells promote peripheral nerve regeneration in vivo without differentiation into Schwann-like lineage. Plast. Reconstr. Surg. 2016, 137 (2), 318e-330e, DOI: 10.1097/01.prs.0000475762.86580.36.

(44) Wang, Y.; Zhao, Z.; Ren, Z.; Zhao, B.; Zhang, L.; Chen, J.; Xu, W.; Lu, S.; Zhao, Q.; Peng, J., Recellularized nerve allografts with differentiated mesenchymal stem cells promote peripheral nerve regeneration. Neurosci. Lett. 2012, 514 (1), 96-101, DOI: 10.1016/j.neulet.2012.02.066. 
(45) di Summa, P. G.; Kingham, P. J.; Raffoul, W.; Wiberg, M.; Terenghi, G.; Kalbermatten, D. F., Adipose-derived stem cells enhance peripheral nerve regeneration. J. Plast. Reconstr. Aesthet. Surg. 2010, 63 (9), 1544-1552, DOI: 10.1016/j.bjps.2009.09.012.

(46) Lopatina, T.; Kalinina, N.; Karagyaur, M.; Stambolsky, D.; Rubina, K.; Revischin, A.; Pavlova, G.; Parfyonova, Y.; Tkachuk, V., Adipose-derived stem cells stimulate regeneration of peripheral nerves: BDNF secreted by these cells promotes nerve healing and axon growth de novo. PloS one 2011, 6 (3), e17899, DOI: 10.1371/journal.pone.0017899.

(47) Xu, Y.; Zhang, Z.; Chen, X.; Li, R.; Li, D.; Feng, S., A silk fibroin/collagen nerve scaffold seeded with a co-culture of schwann cells and adipose-derived stem cells for sciatic nerve regeneration. PloS one 2016, 11 (1), e0147184, DOI: 10.1371/journal.pone.0147184.

(48) Carriel, V.; Garrido-Gómez, J.; Hernández-Cortés, P.; Garzón, I.; García-García, S.; SáezMoreno, J. A.; del Carmen Sanchez-Quevedo, M.; Campos, A.; Alaminos, M., Combination of fibrin-agarose hydrogels and adipose-derived mesenchymal stem cells for peripheral nerve regeneration. J. Neural. Eng. 2013, 10 (2), 026022, DOI: 10.1088/1741-2560/10/2/026022.

(49) Boecker, A. H.; van Neerven, S. G. A.; Scheffel, J.; Tank, J.; Altinova, H.; Seidensticker, K.; Deumens, R.; Tolba, R.; Weis, J.; Brook, G. A., Pre-differentiation of mesenchymal stromal cells in combination with a microstructured nerve guide supports peripheral nerve regeneration in the rat sciatic nerve model. Eur. J. Neurosci. 2016, 43 (3), 404-416, DOI: 10.1111/ejn.13052.

(50) Fu, X.; Tong, Z.; Li, Q.; Niu, Q.; Zhang, Z.; Tong, X.; Tong, L.; Zhang, X., Induction of adipose-derived stem cells into Schwann-like cells and observation of Schwann-like cell proliferation. Mol. Med. Rep. 2016, 14 (2), 1187-1193, DOI: 10.3892/mmr.2016.5367.

(51) Sharma, A. D.; Zbarska, S.; Petersen, E. M.; Marti, M. E.; Mallapragada, S. K.; Sakaguchi, D. S., Oriented growth and transdifferentiation of mesenchymal stem cells towards a Schwann cell fate on micropatterned substrates. J. Biosci. Bioeng. 2016, 121 (3), 325-335, DOI: 10.1016/j.jbiosc.2015.07.006.

(52) Martens, W.; Sanen, K.; Georgiou, M.; Struys, T.; Bronckaers, A.; Ameloot, M.; Phillips, J.; Lambrichts, I., Human dental pulp stem cells can differentiate into Schwann cells and promote and guide neurite outgrowth in an aligned tissue-engineered collagen construct in vitro. FASEB J. 2014, 28 (4), 1634-1643, DOI: 10.1096/fj.13-243980.

(53) Duan, B.; Yin, Z.; Kang, L. H.; Magin, R. L.; Butcher, J. T., Active tissue stiffness modulation controls valve interstitial cell phenotype and osteogenic potential in $3 \mathrm{D}$ culture. Acta biomater. 2016, 36, 42-54, DOI: 10.1016/j.actbio.2016.03.007.

(54) Duan, B.; Hockaday, L. A.; Das, S.; Xu, C.; Butcher, J. T., Comparison of Mesenchymal Stem Cell Source Differentiation Toward Human Pediatric Aortic Valve Interstitial Cells within 3D Engineered Matrices. Tissue Eng. Part C-Methods 2015, 21 (8), 795-807, DOI: 10.1089/ten.tec.2014.0589.

(55)Wu, S.; Wang, Y.; Streubel, P. N.; Duan, B., Living nanofiber yarn-based woven biotextiles for tendon tissue engineering using cell tri-culture and mechanical stimulation. Acta biomater. 2017, 62, 102-115, DOI: 10.1016/j.actbio.2017.08.043. 
(56)Wu, S.; Duan, B.; Liu, P.; Zhang, C.; Qin, X.; Butcher, J. T., Fabrication of aligned nanofiber polymer yarn networks for anisotropic soft tissue scaffolds. ACS Appl. Mater. Interfaces 2016, 8 (26), 16950-16960, DOI: 10.1021/acsami.6b05199.

(57)Wu, S.; Xu, R.; Duan, B.; Jiang, P., Three-dimensional hyaluronic acid hydrogel-based models for in vitro human iPSC-derived NPC culture and differentiation. J. Mater. Chem. B 2017, 5 (21), 3870-3878, DOI: 10.1039/C7TB00721C.

(58)De Luca, A.; Raffoul, W.; Giacalone, F.; Bertolini, M.; Di Summa, P., Tissue-engineered constructs for peripheral nerve repair: current research concepts and future perspectives. Plast. Aesthet. Res. 2015, 2 (4), 213-213, DOI: 10.4103/2347-9264.160889.

(59) Sarker, M.; Saman, N.; McInnes, A. D.; Schreyer, D. J.; Xiongbiao, C., Regeneration of peripheral nerves by nerve guidance conduits: Influence of design, biopolymers, cells, growth factors, and physical stimuli. Prog. Neurobiol. 2018, 171, 125-150, DOI: 10.1016/j.pneurobio.2018.07.002.

(60)Huang, C.; Ouyang, Y.; Niu, H.; He, N.; Ke, Q.; Jin, X.; Li, D.; Fang, J.; Liu, W.; Fan, C., Nerve guidance conduits from aligned nanofibers: improvement of nerve regeneration through longitudinal nanogrooves on a fiber surface. ACS Appl. Mater. Interfaces 2015, 7 (13), 7189-7196, DOI: $10.1021 / \mathrm{am} 509227 \mathrm{t}$.

(61)Zhang, D.; Wu, S.; Feng, J.; Duan, Y.; Xing, D.; Gao, C., Micropatterned biodegradable polyesters clicked with CQAASIKVAV promote cell alignment, directional migration, and neurite outgrowth. Acta biomater. 2018, 74, 143-155, DOI: 10.1016/j.actbio.2018.05.018.

(62)Gu, X.; Ding, F.; Yang, Y.; Liu, J., Construction of tissue engineered nerve grafts and their application in peripheral nerve regeneration. Prog. Neurobiol. 2011, 93 (2), 204-230, DOI: 10.1016/j.pneurobio.2010.11.002.

(63)Novikova, L. N.; Kolar, M. K.; Kingham, P. J.; Ullrich, A.; Oberhoffner, S.; Renardy, M.; Doser, M.; Müller, E.; Wiberg, M.; Novikov, L. N., Trimethylene carbonate-caprolactone conduit with poly-p-dioxanone microfilaments to promote regeneration after spinal cord injury. Acta biomater. 2018, 66, 177-191, DOI: 10.1016/j.actbio.2017.11.028.

(64)Kim, Y. P.; Lee, G. S.; Kim, J. W.; Kim, M. S.; Ahn, H. S.; Lim, J. Y.; Kim, H. W.; Son, Y. J.; Knowles, J. C.; Hyun, J. K., Phosphate glass fibres promote neurite outgrowth and early regeneration in a peripheral nerve injury model. J. Tissue Eng. Regen. Med. 2015, 9 (3), 236-246, DOI: $10.1002 /$ term.1626.

(65)Wang, J.; Tian, L.; Luo, B.; Ramakrishna, S.; Kai, D.; Loh, X. J.; Yang, I. H.; Deen, G. R.; Mo, X., Engineering PCL/lignin nanofibers as an antioxidant scaffold for the growth of neuron and Schwann cell. Colloids Surf. $B$ Biointerfaces 2018, 169, 356-365, DOI: 10.1016/j.colsurfb.2018.05.021.

(66)Neal, R. A.; Tholpady, S. S.; Foley, P. L.; Swami, N.; Ogle, R. C.; Botchwey, E. A., Alignment and composition of laminin-polycaprolactone nanofiber blends enhance peripheral nerve regeneration. J. Biomed. Mater. Res. A 2012, 100 (2), 406-423, DOI: 10.1002/jbm.a.33204. 
(67)Rinoldi, C.; Fallahi, A.; Yazdi, I. K.; Paras, J. C.; Kijenska-Gawronska, E.; Trujillo-de Santiago, G.; Tuoheti, A.; Demarchi, D.; Annabi, N.; Khademhosseini, A.; Swieszkowski, W.; Tamayol, A., Mechanical and Biochemical Stimulation of 3D Multilayered Scaffolds for Tendon Tissue Engineering. ACS Biomater. Sci. Eng. 2019, 5 (6), 2953-2964, DOI: 10.1021/acsbiomaterials.8b01647.

(68)Gnavi, S.; Fornasari, B.; Tonda-Turo, C.; Ciardelli, G.; Zanetti, M.; Geuna, S.; Perroteau, I., The influence of electrospun fibre size on Schwann cell behaviour and axonal outgrowth. Mater. Sci. Eng. C 2015, 48, 620-631, DOI: 10.1016/j.msec.2014.12.055.

(69)Zhu, W.; Masood, F.; O'Brien, J.; Zhang, L. G., Highly aligned nanocomposite scaffolds by electrospinning and electrospraying for neural tissue regeneration. Nanomed.-Nanotechnol. Biol. Med. 2015, 11 (3), 693-704, DOI: 10.1016/j.nano.2014.12.001.

(70)Daniele, M. A.; Boyd, D. A.; Adams, A. A.; Ligler, F. S., Microfluidic Strategies for Design and Assembly of Microfibers and Nanofibers with Tissue Engineering and Regenerative Medicine Applications. Adv. Healthc. Mater. 2015, 4 (1), DOI: 10.1002/adhm.201400144.

(71)Neibert, K.; Gopishetty, V.; Grigoryev, A.; Tokarev, I.; Al-Hajaj, N.; Vorstenbosch, J.; Philip, A.; Minko, S.; Maysinger, D., Wound-Healing with Mechanically Robust and Biodegradable Hydrogel Fibers Loaded with Silver Nanoparticles. Adv. Healthc. Mater. 2012, 1 (5), 621-630, DOI: 10.1002/adhm.201200075.

(72)Akbari, M.; Tamayol, A.; Laforte, V.; Annabi, N.; Najafabadi, A. H.; Khademhosseini, A.; Juncker, D., Composite Living Fibers for Creating Tissue Constructs Using Textile Techniques. Adv. Funct. Mater. 2014, 24 (26), 4060-4067, DOI: 10.1002/adfm.201303655.

(73)Tajdaran, K.; Chan, K.; Gordon, T.; Borschel, G. H., Matrices, scaffolds, and carriers for protein and molecule delivery in peripheral nerve regeneration. Exp. Neurol. 2019, 319, 112817112817, DOI: 10.1016/j.expneurol.2018.08.014.

(74)Lu, J.; Sun, X.; Yin, H.; Shen, X.; Yang, S.; Wang, Y.; Jiang, W.; Sun, Y.; Zhao, L.; Sun, X.; Lu, S.; Mikos, A. G.; Peng, J.; Wang, X., A neurotrophic peptide-functionalized self-assembling peptide nanofiber hydrogel enhances rat sciatic nerve regeneration. Nano Res. 2018, 11 (9), 45994613, DOI: 10.1007/s12274-018-2041-9.

(75)Tamayol, A.; Najafabadi, A. H.; Aliakbarian, B.; Arab-Tehrany, E.; Akbari, M.; Annabi, N.; Juncker, D.; Khademhosseini, A., Hydrogel Templates for Rapid Manufacturing of Bioactive Fibers and 3D Constructs. Adv. Healthc. Mater. 2015, 4 (14), 2146-2153, DOI: 10.1002/adhm.201500492.

(76)Gu, X.; Ding, F.; Williams, D. F., Neural tissue engineering options for peripheral nerve regeneration. Biomaterials 2014, 35 (24), 6143-6156, DOI: 10.1016/j.biomaterials.2014.04.064.

(77)Khorshidi, S.; Solouk, A.; Mirzadeh, H.; Mazinani, S.; Lagaron, J. M.; Sharifi, S.; Ramakrishna, S., A review of key challenges of electrospun scaffolds for tissue - engineering applications. J. Tissue Eng. Regen. Med. 2016, 10 (9), 715-738, DOI: 10.1002/term.1978. 
(78)Haider, A.; Haider, S.; Kang, I. K., A comprehensive review summarizing the effect of electrospinning parameters and potential applications of nanofibers in biomedical and biotechnology. Arab. J. Chem. 2018, 11, 1165, DOI: 10.1016/j.arabjc.2015.11.015.

(79)Cirillo, V.; Bushman, J.; Guarino, V.; Kohn, J.; Ambrosio, L., 3D conduits for peripheral nerve regeneration. Electrofluidodynamic Technologies (EFDTs) for Biomaterials and Medical Devices, Elsevier: 2018, pp 329-349, DOI: 10.1016/B978-0-08-101745-6.00016-5.

(80)Jessen, K.; Mirsky, R., The repair Schwann cell and its function in regenerating nerves. $J$. Physiol. 2016, 594 (13), 3521-3531, DOI: 10.1113/JP270874.

(81)Ning, L.; Xu, Y.; Chen, X.; Schreyer, D. J., Influence of mechanical properties of alginatebased substrates on the performance of Schwann cells in culture. J. Biomater. Sci. Polym. Ed. 2016, 27 (9), 898-915, DOI: 10.1080/09205063.2016.1170415.

(82)Bayat, N.; Ebrahimi-Barough, S.; Ardakan, M. M. M.; Ai, A.; Kamyab, A.; Babaloo, N.; Ai, J., Differentiation of human endometrial stem cells into Schwann cells in fibrin hydrogel as 3D culture. Mol. Neurobiol. 2016, 53 (10), 7170-7176, DOI: 10.1007/s12035-015-9574-7.

(83)Sun, X.; Zhu, Y.; Yin, H. Y.; Guo, Z. Y.; Xu, F.; Xiao, B.; Jiang, W. L.; Guo, W. M.; Meng, H. Y.; Lu, S. B., Differentiation of adipose-derived stem cells into Schwann cell-like cells through intermittent induction: potential advantage of cellular transient memory function. Stem Cell Res. Ther. 2018, 9 (1), 133, DOI: 10.1186/s13287-018-0884-3.

(84)Liu, Q.; Spusta, S. C.; Mi, R.; Lassiter, R. N.; Stark, M. R.; Höke, A.; Rao, M. S.; Zeng, X., Human neural crest stem cells derived from human ESCs and induced pluripotent stem cells: induction, maintenance, and differentiation into functional schwann cells. Stem Cell Transl. Med. 2012, 1 (4), 266-278, DOI: 10.5966/sctm.2011-0042.

(85)Uz, M.; Büyüköz, M.; Sharma, A. D.; Sakaguchi, D. S.; Altinkaya, S. A.; Mallapragada, S. K., Gelatin-based 3D conduits for transdifferentiation of mesenchymal stem cells into Schwann cell-like phenotypes. Acta biomater. 2017, 53, 293-306, DOI: 10.1016/j.actbio.2017.02.018.

(86)Xue, J.; Yang, J.; O’Connor, D. M.; Zhu, C.; Huo, D.; Boulis, N. M.; Xia, Y., Differentiation of Bone Marrow Stem Cells into Schwann Cells for the Promotion of Neurite Outgrowth on Electrospun Fibers. ACS Appl. Mater. Interfaces 2017, 9 (14), 12299-12310, DOI: 10.1021/acsami.7b00882.

(87)Ebrahimi-Barough, S.; Javidan, A. N.; Saberi, H.; Joghataei, M. T.; Rahbarghazi, R.; Mirzaei, E.; Faghihi, F.; Shirian, S.; Ai, A.; Ai, J., Evaluation of motor neuron-like cell differentiation of hEnSCs on biodegradable PLGA nanofiber scaffolds. Mol. Neurobiol. 2015, 52 (3), 1704-1713, DOI: 10.1007/s12035-014-8931-2.

(88) Jessen, K. R.; Mirsky, R.; Lloyd, A. C., Schwann cells: development and role in nerve repair. Cold Spring Harb. Perspect. Biol. 2015, a020487, DOI: 10.1101/cshperspect.a020487.

(89)Boerboom, A.; Dion, V.; Chariot, A.; Franzen, R., Molecular mechanisms involved in Schwann cell plasticity. Front. Mol. Neurosci. 2017, 10, 38, DOI: 10.3389/fnmol.2017.00038. 


\section{For Table of Contents Use Only}

Guiding Mesenchymal Stem Cells into Myelinating Schwann Cell-like Phenotypes by Using

\section{Electrospun Core-Sheath Nanoyarns}

Shaohua Wu, Shilei Ni, Xiping Jiang, Mitchell A. Kuss, Hanjun Wang, Bin Duan*

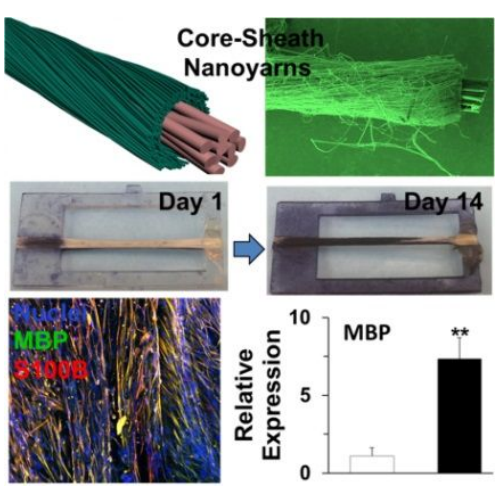

